J. Lake Sci. (湖泊科学), 2012, 24(2):259-266

http: //www. jlakes.org. E-mail : jlakes@niglas.ac.cn

(C) 2012 by Journal of Lake Sciences

\title{
江苏省西部湖泊溶解性有机物光谱学特征和来源解析”
}

\author{
陈小锋, 揣小明, 刘 涛, 杨柳燕 ${ }^{* *}$ \\ （南京大学环境学院污染控制与资源化研究国家重点实验室,南京 210046）
}

\begin{abstract}
摘 要: 利用光谱学手段研究江苏省西部湖泊表层水体中溶解性有机物 (DOM) 组成与结构,并对其来源进行分析. 单位 浓度可溶性有机碳在 254 和 $280 \mathrm{~nm}$ 波长下的吸光度值 $(S U V A)$ 测定结果表明, 各湖泊芳香性程度及分子量大小依次为邵 伯湖 $>$ 天岗湖 $>$ 白马湖 $>$ 石臼湖 $>$ 洪泽湖 $>$ 固城湖 $>$ 骆马湖 $>$ 高邮湖 $>$ 宝应湖. 特定波长下吸光度的比值 $\left(E_{2} / E_{3} 、 E_{3} /\right.$ $\left.E_{4}\right)$ 显示邵伯湖和白马湖中的 DOM 结构复杂、分子量大、苯环多, 以腐殖酸为主要成分; 其它湖泊的 DOM 腐殖化程度较 低, 以富里酸为主. 指数函数曲线斜率 $\left(S_{275} \sim 295 \mathrm{~nm}\right)$ 拟合结果也同样表明邵伯湖 DOM 分子量最大, 而宝应湖最低. 各湖泊 荧光指数和生物指数分别处于 $1.13 \sim 1.30$ 和 $0.47 \sim 0.67$ 范围内, 体现出 DOM 强烈的陆源性. 四个主要苂光峰的相对苂 光强度之间均存在良好的相关性, 表明这些湖泊的类腐殖酸及类蛋白物质可能有着相同的来源. 结合这些湖泊的特征及 流域经济发展水平, 可以初步推断经人湖河流携带的由农业及其下游产业产生的有机质是江苏西部湖泊中 DOM 的主要 来源.
\end{abstract}

关键词: 湖泊; 溶解性有机物; 光谱学;江苏省

\section{Characteristics and source identification of the dissolved organic matter in the lakes of west Jiangsu by spectroscopy}

\section{CHEN Xiaofeng, CHUAI Xiaoming, LIU Tao \& YANG Liuyan}

(State Key Laboratory of Pollution Control and Resource Reuse, School of the Environment, Nanjing University, Nanjing 210046, P. R. China)

Abstract: The composition and origin of the dissolved organic matter (DOM) in some lakes of west Jiangsu was investigated using UV-Vis absorbance and fluorescence spectroscopy. The specific ultraviolet absorption (SUVA) at $254 \mathrm{~nm}$ and $280 \mathrm{~nm}$ indicated that the aromaticity and molecular weight of DOM ranked in the order of Lake Shaobo, Lake Tiangang, Lake Baima, Lake Shijiu, Lake Hongze, Lake Gucheng, Lake Luoma, Lake Gaoyou and Lake Baoying. The $E_{2} / E_{3}$ and $E_{3} / E_{4}$ values suggested that the DOM of Lake Shaobo and Lake Baima was structural complex with humic acid being the primary component, while other lakes' DOM was less complex and constituted mostly by fulvic acid. The exponential slope parameter $\left(S_{275-295 \mathrm{~nm}}\right)$ values results were consistent with the conclusion obtained from $S U V A, E_{2} / E_{3}$ and $E_{3} / E_{4}$. Fluorescence indexes and biological index of these lakes' DOM were ranged of $1.13-1.30,0.47-0.67$, respectively, suggesting DOM a strong terrestrial origin. Significant correlation was found between the fluorescence intensities per unit organic carbon at different peaks, which indicated that the humic-like and protein-like materials in these lakes have a similar source. Considered the characterization of these lakes and the economic development, it can be deduced that most of these DOM are produced by agriculture and its downstream industries and imported with inflow rivers.

Keywords: Lake; dissolved organic matter; spectroscopy; Jiangsu Province

溶解性有机质 (DOM) 是指存在于各种天然水体中、可以通过 $0.45 \mu \mathrm{m}$ 或 $0.7 \mu \mathrm{m}$ 滤膜的天然有机质混 合体,其组分包括腐殖酸、富里酸以及各种亲水性有机酸、羧酸、氨基酸、碳水化合物等 ${ }^{[1]}$,在水生态系统营

* 国家水体污染控制与治理科技重大专项项目 (2009ZX07106-001-001) 资助. 2011-03-24 收稿;2011-10-10 收修 改稿. 陈小锋,男,1975 年生,博士研究生;E-mail:xfchen@yzu. edu.cn.

** 通信作者; E-mail : yangly@ nju. edu. cn. 
养物质的生物化学循环过程中起着相当重要的作用 ${ }^{[2-4]}$. 对水域中 DOM 组成和来源进行研究, 有助于认识 水生态系统中营养物质生物化学循环的特征, 从而为水生态系统的保护提供依据. 由于在水生态系统中的 重要作用及化学组成和结构的复杂性, DOM 成为近三十年来水环境研究的热点问题之一. 目前, 对 DOM 进 行分析的手段很多, 包括天然稳定性同位素技术 ${ }^{[5-6]}$ 、超滤 ${ }^{[7-8]}$ 、凝胶渗透色谱 ${ }^{[9-10]}$ 和三维苂光光谱 ${ }^{[11]}$ 等. 相 对于其它方法, 光谱学测试具有样品预处理简单、操作简便、灵敏度高等优点, 因此被越来越广泛地用于天 然水体 DOM 光谱特征、分子量特征分析和物质组成及来源鉴别 ${ }^{[12-14]}$.

江苏省是我国淡水湖泊分布最集中的省区之一,境内有大小湖荡 200 余个,湖泊率达 $6 \%$, 居全国首 位 ${ }^{[15]}$. 但是近 $30 \mathrm{a}$ 来, 工业和生活污水的排放、土地的高强度利用等人类活动使得江苏地区河湖水系逐步 遭受污染威胁 ${ }^{[16]}$, 因此本研究利用紫外-可见光谱及三维苂光技术分析江苏西部 9 个湖泊 DOM 的组成特 征,并对其来源进行探讨.

\section{1 材料和方法}

\section{1 样品采集与处理}

2010 年 6 月,在固城湖及石臼湖 (图 1) 用有机玻璃采水器采集 $1 \mathrm{~L}$ 表层 $0.5 \mathrm{~m}$ 处水样,用鲁哥氏液现场 固定, 静置 $48 \mathrm{~h}$ 浓缩至 $30 \mathrm{ml}$ 后, 用浮游植物计数框在光学显微镜 $(\times 40)$ 下观察计数 ${ }^{[17]} .2010$ 年 10 月在江 苏西部 9 个湖泊 (图 1) 中, 根据湖泊大小及功能布点采集表层 $(0 \sim 0.5 \mathrm{~m}$ ) 水样, 同时用 YSI 6600 多功能水 质仪测定水体温度、 $\mathrm{pH}$ 、溶解氧、电导率等物理化学参数. 采集的水样置于冷藏箱内低温保存, 运回实验室后 立即用 $0.7 \mu \mathrm{m}$ 玻璃纤维滤膜 (预先 $450^{\circ} \mathrm{C}$ 灼烧) 过滤, 测定前置冰箱中 $4^{\circ} \mathrm{C}$ 保存.

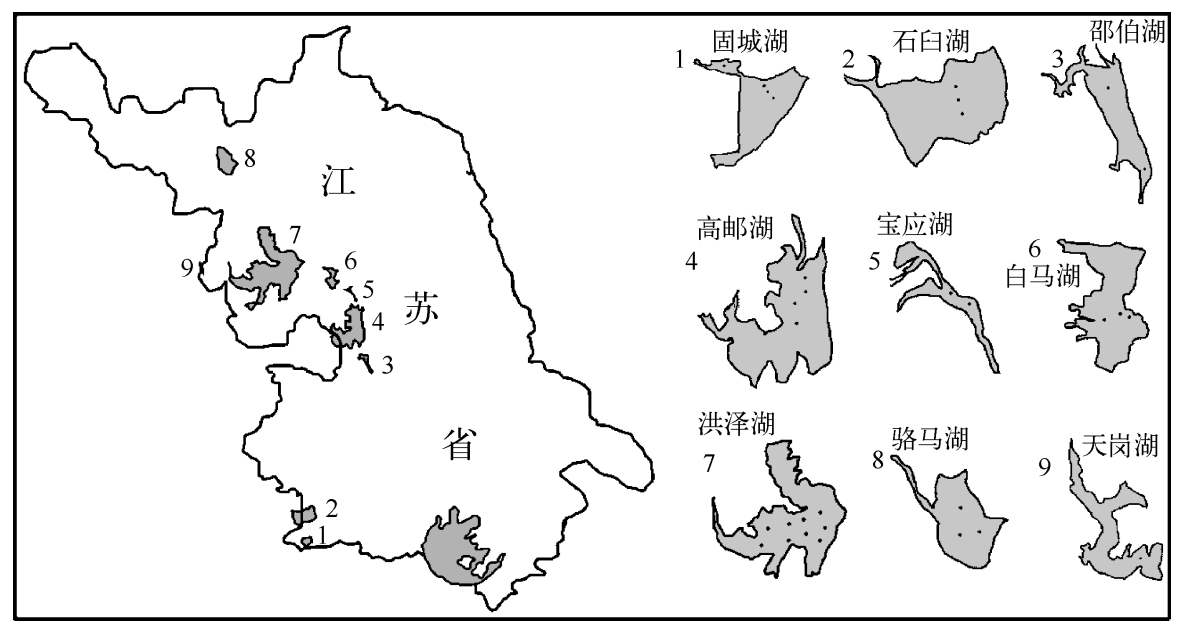

图 1 江苏省西部湖泊采样位点图

Fig. 1 Location of sampling lakes in Jiangsu Province

\section{2 样品分析}

已过滤的水样用日本岛津 TOC5000A 总有机碳测定仪测定可溶性有机碳 (DOC) 含量, 仪器检测限为 $0.004 \mathrm{mg} / \mathrm{L}$, 标准偏差 $<2 \%$. 过滤后的水样置于 $1 \mathrm{~cm}$ 石英比色具中, 采用岛津 2450 紫外-可见分光光度计 测定 $200 \sim 600 \mathrm{~nm}$ 吸收光谱, 扫描间距 $0.5 \mathrm{~nm}$. 为了消除过滤清液中残留细小颗粒物的散射, 利用 $700 \mathrm{~nm}$ 处 吸收系数进行散射效应订正 ${ }^{[18]}$.

三维苂光光谱用 HitachiF-7000 型荧光分光光度计测定, 仪器光源为 $150 \mathrm{~W}$ 氙灯, 光电倍增管电压 $700 \mathrm{~V}$, 激发 $(E x)$ 和发射 $(E m)$ 单色器均为衍射光栅, 波长误差 $\pm 2 \mathrm{~nm}$, 激发波长为 $350 \mathrm{~nm}$ 时水的拉曼峰信躁比 $S / N>250(\mathrm{P}-\mathrm{P})$. 激发和发射狭缝宽度均为 $5 \mathrm{~nm}$, 扫描波长范围 $\lambda_{\mathrm{Ex}}$ 为 $200 \sim 450 \mathrm{~nm}, \lambda_{\mathrm{Em}}$ 为 $250 \sim 600 \mathrm{~nm}$, 步 长 $5 \mathrm{~nm}$, 扫描速度 $1200 \mathrm{~nm} / \mathrm{min}$. 为消除水 Rayleigh 和 Raman 散射的影响, 将图谱中 $E x \geqslant E m, 2 \times E x \leqslant E m$ 及 $E x=E m \pm 10 \sim 15 \mathrm{~nm}$ 等区域中的数据以 0 代替 ${ }^{[19-20]}$. 利用紫外-可见扫描数据对所得苂光值进行内滤波效应 
校正 ${ }^{[21]}$, 同时为消除仪器间差别, 采用 $0.01 \mathrm{mg} / \mathrm{L}$ 硫酸奎宁的稀硫酸硫酸溶液在 $E x / E m=350 \mathrm{~nm} / 450 \mathrm{~nm}$ 处 的苂光强度将所测苂光强度换算成标准苂光强度后进行比较 ${ }^{[13]}$. 处理后数据采用 Sigmaplot 11 绘制等高图.

\section{3 单位 DOC 浓度样品的吸收系数及指数函数斜率的计算}

单位 DOC 浓度的样品在特定波长下的吸收系数 $(S U V A)$ 采用公式 $S U V A=A / b c$ 进行计算 ${ }^{[22]}$, 式中, $A$ 是 该波长下的吸收值, $b$ 为比色血光程 $(\mathrm{m}), c$ 为可溶性有机碳的浓度 $(\mathrm{mg} / \mathrm{L})$.

DOM 光谱吸收基本上呈现指数衰减的规律, Bricaud 等 ${ }^{[23]}$ 提出如下公式: $a(\lambda)=a\left(\lambda_{0}\right) \exp \left[S\left(\lambda_{0}-\lambda\right)\right]$; 式中, $a(\lambda)$ 为 DOM 的吸收系数 $\left(\mathrm{m}^{-1}\right) ; \lambda$ 为波长 $(\mathrm{nm}) ; \lambda_{0}$ 为参照波长 $(\mathrm{nm}) ; S$ 为指数函数曲线斜率参数 $\left(\mu \mathrm{m}^{-1}\right)$, 在 $275 \sim 295 \mathrm{~nm}$ 波段采用线性拟合获得.

\section{4 数据处理}

采用 SPSS 13 对数据进行统计和分析,包括线性拟合及相关性分析等.

\section{2 结果与讨论}

\section{1 湖泊基本水质参数}

2010 年 10 月江苏省西部湖泊的基本水质参数可以看出,9 个湖泊水体均呈弱碱性 ( pH 7.4 8.5), 溶 解氧除白马湖 $(4.76 \mathrm{mg} / \mathrm{L}$ ) 外, 其它湖泊相差不大. 而各湖泊的 DOM 浓度则有着较大的差异, 其中宝应湖的 DOM 浓度最高 (以 C 计), 达到 $23.08 \mathrm{mg} / \mathrm{L}$, 而邵伯湖的 DOM 浓度只有 $6.85 \mathrm{mg} / \mathrm{L}$ (表 1 ).

表 1 江苏省西部湖泊的基本水质参数

Tab. 1 The physical and chemical properties of the lake water in the west of Jiangsu Province

\begin{tabular}{ccccccc}
\hline 湖泊 & 温度 $/{ }^{\circ} \mathrm{C}$ & $\mathrm{pH}$ & 溶解氧 $/(\mathrm{mg} / \mathrm{L})$ & 电导率 $/(\mu \mathrm{S} / \mathrm{cm})$ & 盐度 $/(\mathrm{g} / \mathrm{L})$ & $\mathrm{DOC} /(\mathrm{mg} / \mathrm{L})$ \\
\hline 固城湖 & $16.27 \pm 0.06$ & $7.95 \pm 0.16$ & $9.71 \pm 0.41$ & $0.23 \pm 0.01$ & $0.11 \pm 0.01$ & $8.34 \pm 1.52$ \\
石臼湖 & $14.75 \pm 0.01$ & $7.79 \pm 0.50$ & $11.31 \pm 0.55$ & $0.24 \pm 0.00$ & $0.11 \pm 0.00$ & $8.73 \pm 0.79$ \\
邵伯湖 & $19.90 \pm 0.20$ & $7.72 \pm 0.16$ & $7.69 \pm 0.31$ & $0.47 \pm 0.02$ & $0.23 \pm 0.01$ & $6.85 \pm 0.69$ \\
高邮湖 & $18.83 \pm 0.09$ & $8.50 \pm 0.21$ & $9.89 \pm 0.45$ & $0.43 \pm 0.01$ & $0.21 \pm 0.01$ & $15.89 \pm 1.51$ \\
宝应湖 & $19.44 \pm 0.19$ & $8.20 \pm 0.04$ & $9.94 \pm 0.37$ & $0.49 \pm 0.00$ & $0.24 \pm 0.00$ & $23.08 \pm 1.27$ \\
白马湖 & $19.40 \pm 0.14$ & $7.40 \pm 0.06$ & $4.76 \pm 0.18$ & $0.46 \pm 0.02$ & $0.22 \pm 0.01$ & $11.74 \pm 0.76$ \\
洪泽湖 & $18.28 \pm 0.09$ & $8.25 \pm 0.10$ & $9.37 \pm 0.05$ & $0.41 \pm 0.02$ & $0.20 \pm 0.01$ & $12.21 \pm 1.13$ \\
骆马湖 & $18.69 \pm 0.05$ & $8.30 \pm 0.04$ & $9.10 \pm 0.14$ & $0.91 \pm 0.00$ & $0.45 \pm 0.00$ & $14.24 \pm 1.27$ \\
天岗湖 & 19.10 & 7.96 & 8.95 & 0.56 & 0.27 & 10.91 \\
\hline
\end{tabular}

\subsection{DOM 的组成结构分析}

紫外-可见吸收光谱(UV-Vis Absorbance Spectroscopy) 已经广泛地应用于水、土壤中可溶性有机物的表 征研究 ${ }^{[24]}$. 特定波长下紫外吸收值对可溶性有机碳的比值 (SUVA) 可用来区分可溶性有机物组成 ${ }^{[25]}$. 如 $S U V A_{254 \mathrm{~mm}}$ 常被用于表征腐殖质样品中的芳香性结构, 其值越高芳香性越强 ${ }^{[26]}$. 而 Chin 等 ${ }^{[27]}$ 则认为酚类、苯 胺衍生物、安息香酸、多烯等物质在 $270 \sim 280 \mathrm{~nm}$ 发生 $\pi \sim \pi^{*}$ 电子迁移, 因此选择 $280 \mathrm{~nm}$ 波长下的吸光度 值进行研究, 结果发现 $S U V A_{280 \mathrm{~mm}}$ 可以提供可溶性有机质的芳香性程度、源、腐殖化程度和分子量等重要的信 息, 并且该值与 DOM 的芳香性、平均分子量均具有良好的相关性. 特定波长的 UV-Vis 吸光值比也可用来指 示水溶性有机物的组成、团聚化程度和分子质量的大小 ${ }^{[28]} . E_{2} / E_{3}$ 是 250 和 $365 \mathrm{~nm}$ 处的紫外吸收值之比, 有 研究认为 $E_{2} / E_{3}$ 可以较好地反映水溶性有机质的分子状况, $E_{2} / E_{3}$ 越小, 则水溶性有机物的分子质量越 $大^{[29]}$. Peuravuori 等 ${ }^{[30]}$ 发现 $E_{2} / E_{3}$ 可作为湖沼中腐殖化的标志物, 通常来说, 腐殖酸相对于富里酸具有高的 $S U V A_{280 \mathrm{~mm}}$ 和低的 $E_{2} / E_{3}$. Abbt-Braun 等 ${ }^{[31]}$ 则选择 300 和 $400 \mathrm{~nm}$ 处的紫外吸收值之比 $\left(E_{3} / E_{4}\right)$ 进行研究, 他们 认为 $E_{3} / E_{4}<3.5$ 时湖泊中水溶性有机物以腐殖酸为主, 而 $E_{3} / E_{4}>3.5$ 时则主要是富里酸. 因此, 本研究采 用紫外-可见吸收光谱对江苏西部湖泊 DOM 特征进行分析, 了解其化学组成.

各湖泊样品在一些特定波长下的 SUVA 值表明, 在波长为 254 和 $280 \mathrm{~nm}$ 时, 各湖泊水样的 SUVA 值依次 
为邵伯湖 $>$ 天岗湖 $>$ 白马湖 $>$ 洪泽湖 $>$ 固城湖 $>$ 石臼湖 $>$ 骆马湖 $>$ 高邮湖 $>$ 宝应湖 (表 2), 这说明邵伯湖 中 DOM 的芳香性程度和平均分子量最高而宝应湖最低. 从 $E_{2} / E_{3}$ 值可以看出, 各湖泊的 DOM 中腐殖酸的 比例及 DOM 平均分子量大小依次为宝应湖 $>$ 高邮湖 $>$ 骆马湖 $>$ 固城湖 $>$ 天岗湖 $>$ 石臼湖 $>$ 洪泽湖 $>$ 白马 湖 > 邵伯湖, 与 $S U V A$ 的结果基本一致. 邵伯湖的 $E_{3} / E_{4}$ 值小于 3.5 , 说明蓝移的程度较小, DOM 的结构复 杂、分子量大、苯环多, 腐殖化程度较高, DOM 以腐殖酸为主; 其它湖泊的样品基本保持在 $3.96 \sim 7.00$, 说明 蓝移的程度较大, 腐殖化程度较低, DOM 以富里酸为主, 腐殖酸的含量较少.

指数函数斜率 $S$ 值是表征有色可溶性有机物吸收系数随波长增加而递减的参数 ${ }^{[23]}$, 其数值大小与有机 物结构及拟合时所选取的波段有关. Helms 等 ${ }^{[32]}$ 对指数函数斜率与 DOM 分子量、来源关系进行研究, 发现 $275 \sim 295 \mathrm{~nm}$ 波长范围内拟合所得指数函数斜率 $S_{275 \sim 295 \mathrm{~nm}}$ 值与 DOM 的分子量呈显著负相关, 此后, $S_{275 \sim 295 \mathrm{~nm}}$ 得到广泛的应用. Tamara 等 ${ }^{[33]}$ 发现 Mckenzie 河中 $S_{275 ~ 295 \mathrm{~nm}}$ 值在 11.1 15.5 $\mathrm{mm}^{-1}$ 间变化, 而海水的 $S_{275 ~ 295 \mathrm{~nm}}$ 值为 $13.4 \sim 48.3 \mu \mathrm{m}^{-1[34]}$. 国内张运林等 ${ }^{[35]}$ 对太湖 DOM 吸收的 $S_{275 \sim 295 \mathrm{~nm}}$ 值进行拟合, 其值约为

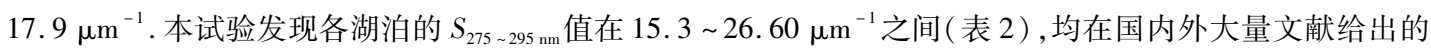
范围内. 比较 $S_{275 ~ 295 n m}$ 值大小可发现郡伯湖 DOM 分子量最大, 而宝应湖最小, 与前面的研究结果一致.

表 2 江苏省西部湖泊的 DOM 光谱学指标

Tab. 2 Some spectroscopic indices of DOM in lakes of west Jiangsu

\begin{tabular}{cccccccccc}
\hline 湖泊 & $\begin{array}{c}S U V A_{254 \mathrm{~nm}} \\
(\mathrm{~L} / \mathrm{mg} \mathrm{C} \cdot \mathrm{m})\end{array}$ & $\begin{array}{c}S U V A_{280 \mathrm{~nm}} \\
(\mathrm{~L} / \mathrm{mg} \mathrm{C} \cdot \mathrm{m})\end{array}$ & $E_{2} / E_{3}{ }^{1)}$ & $E_{3} / E_{4}{ }^{1)}$ & $\begin{array}{c}S_{275} \sim 295 \mathrm{~nm} \\
/\left(\mu \mathrm{m}^{-1}\right)\end{array}$ & $r(\mathrm{~A}, \mathrm{C})$ & $r(\mathrm{~B}, \mathrm{C})$ & $F I$ & $B I X$ \\
\hline 固城湖 & $0.87 \pm 0.18$ & $0.64 \pm 0.14$ & $8.03 \pm 1.60$ & $6.07 \pm 2.04$ & $24.5 \pm 0.95$ & $1.16 \pm 0.09$ & $0.49 \pm 0.03$ & $1.23 \pm 0.07$ & $0.66 \pm 0.06$ \\
石臼湖 & $0.79 \pm 0.07$ & $0.56 \pm 0.05$ & $6.53 \pm 0.39$ & $3.96 \pm 0.47$ & $23.1 \pm 0.14$ & $1.15 \pm 0.01$ & $0.53 \pm 0.03$ & $1.22 \pm 0.06$ & $0.67 \pm 0.02$ \\
邵伯湖 & $2.38 \pm 0.16$ & $1.82 \pm 0.08$ & $4.17 \pm 0.65$ & $3.25 \pm 0.59$ & $15.3 \pm 0.36$ & $1.29 \pm 0.04$ & $0.42 \pm 0.02$ & $1.17 \pm 0.03$ & $0.56 \pm 0.03$ \\
高邮湖 & $0.73 \pm 0.22$ & $0.49 \pm 0.15$ & $9.01 \pm 0.72$ & $6.51 \pm 0.55$ & $26.3 \pm 1.48$ & $1.13 \pm 0.11$ & $0.39 \pm 0.07$ & $1.27 \pm 0.04$ & $0.54 \pm 0.07$ \\
宝应湖 & $0.57 \pm 0.03$ & $0.38 \pm 0.02$ & $9.36 \pm 0.00$ & $6.25 \pm 0.00$ & $26.6 \pm 0.00$ & $1.11 \pm 0.00$ & $0.26 \pm 0.03$ & $1.24 \pm 0.05$ & $0.51 \pm 0.01$ \\
白马湖 & $1.02 \pm 0.12$ & $0.77 \pm 0.04$ & $5.00 \pm 0.49$ & $3.88 \pm 0.37$ & $17.6 \pm 0.27$ & $1.08 \pm 0.04$ & $0.24 \pm 0.03$ & $1.13 \pm 0.03$ & $0.47 \pm 0.02$ \\
洪泽湖 & $1.01 \pm 0.40$ & $0.73 \pm 0.12$ & $6.20 \pm 0.84$ & $4.73 \pm 0.68$ & $20.6 \pm 1.96$ & $1.06 \pm 0.07$ & $0.34 \pm 0.05$ & $1.24 \pm 0.09$ & $0.49 \pm 0.05$ \\
骆马湖 & $0.75 \pm 0.05$ & $0.53 \pm 0.02$ & $8.23 \pm 0.22$ & $6.36 \pm 0.10$ & $24.7 \pm 0.00$ & $1.13 \pm 0.00$ & $0.41 \pm 0.02$ & $1.30 \pm 0.02$ & $0.66 \pm 0.01$ \\
天岗湖 & 1.06 & 0.79 & 6.67 & 7.00 & 19.6 & 0.99 & 0.25 & 1.26 & 0.51 \\
\hline
\end{tabular}

1) $E_{2} / E_{3}$ 和 $E_{3} / E_{4}$ 分别为水样在 250 和 $365 \mathrm{~nm}$ 处吸收值之比和 300 与 $400 \mathrm{~nm}$ 处吸收值之比.

\section{3 湖泊 DOM 源分析}

江苏省西部不同湖泊的 DOM 三维苂光光谱图可以看出, 苂光峰主要有 4 类, 包括 2 个类腐殖酸苂光峰 (A 峰, $E x / E m=230 \sim 260 \mathrm{~nm} / 410 \sim 450 \mathrm{~nm}$; C 峰, $E x / E m=320 \sim 340 \mathrm{~nm} / 430 \sim 450 \mathrm{~nm}$ ) 以及 2 个类蛋白菼光 峰 (B 峰, $E x / E m=280 \mathrm{~nm} / 310 \mathrm{~nm}$; D 峰, $E x / E m=220 \sim 230 \mathrm{~nm} / 310 \sim 330 \mathrm{~nm})^{[11]}$ (图 2). 一般认为 A、C 峰反 映的是外源输人的富里酸和腐殖酸形成的荧光峰值, 与腐殖质结构中的羰基和羧基有关, 而 $\mathrm{B} 、 \mathrm{D}$ 峰则反映 的是生物降解来源的色氨酸和酪氨酸形成的苂光峰值 ${ }^{[11]}$. 三维苂光光谱图中, 苂光峰 $\mathrm{A}$ 和 $\mathrm{C}$ 较强, 而类蛋 白苂光峰 B 和 D 相对较弱, 说明这些湖泊的来源以陆源有机质为主, 由湖泊微生物产生的有机质比较少. 浮 游动植物细胞渗漏、有机体降解等过程 ${ }^{[36-37]}$ 会产生类蛋白物质, 从而导致苂光峰 $\mathrm{B}$ 和 $\mathrm{C}$ 苂光强度的比值 $r(\mathrm{~B}, \mathrm{C})$ 升高, 因此刘明亮等 ${ }^{[38]}$ 提出 $r(\mathrm{~B}, \mathrm{C})$ 可以作为区分 DOM 内外源贡献的重要参数. 西部湖泊 DOM 的

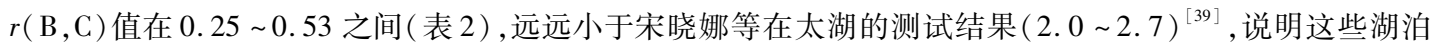
的 DOM 的外源性远远强于太湖. 2010 年 6 月测得石臼湖和固城湖的藻密度约为 $10^{6} \sim 10^{7}$ cells $/ \mathrm{L}$, 相对较高 的浮游植物密度可能是这两个湖泊 $r(\mathrm{~B}, \mathrm{C})$ 值 $(0.49 \sim 0.53)$ 大于其它湖泊的原因.

特定波长苂光强度的比值可以用来区分外源和内源的相对贡献. McKnight 等 $^{[26]}$ 发现激发光波长为 $370 \mathrm{~nm}$ 时,苂光发射光谱强度在 450 与 $500 \mathrm{~nm}$ 处的比值(苂光指数, FI) 可以提供 DOM 来源的信息, 并确定 了陆源和内源的临界值分别为 1.4 和 1.9 . 生物指数 $(B I X)$ 是激发波长为 $310 \mathrm{~nm}$ 时, 荧光发射光谱强度在 


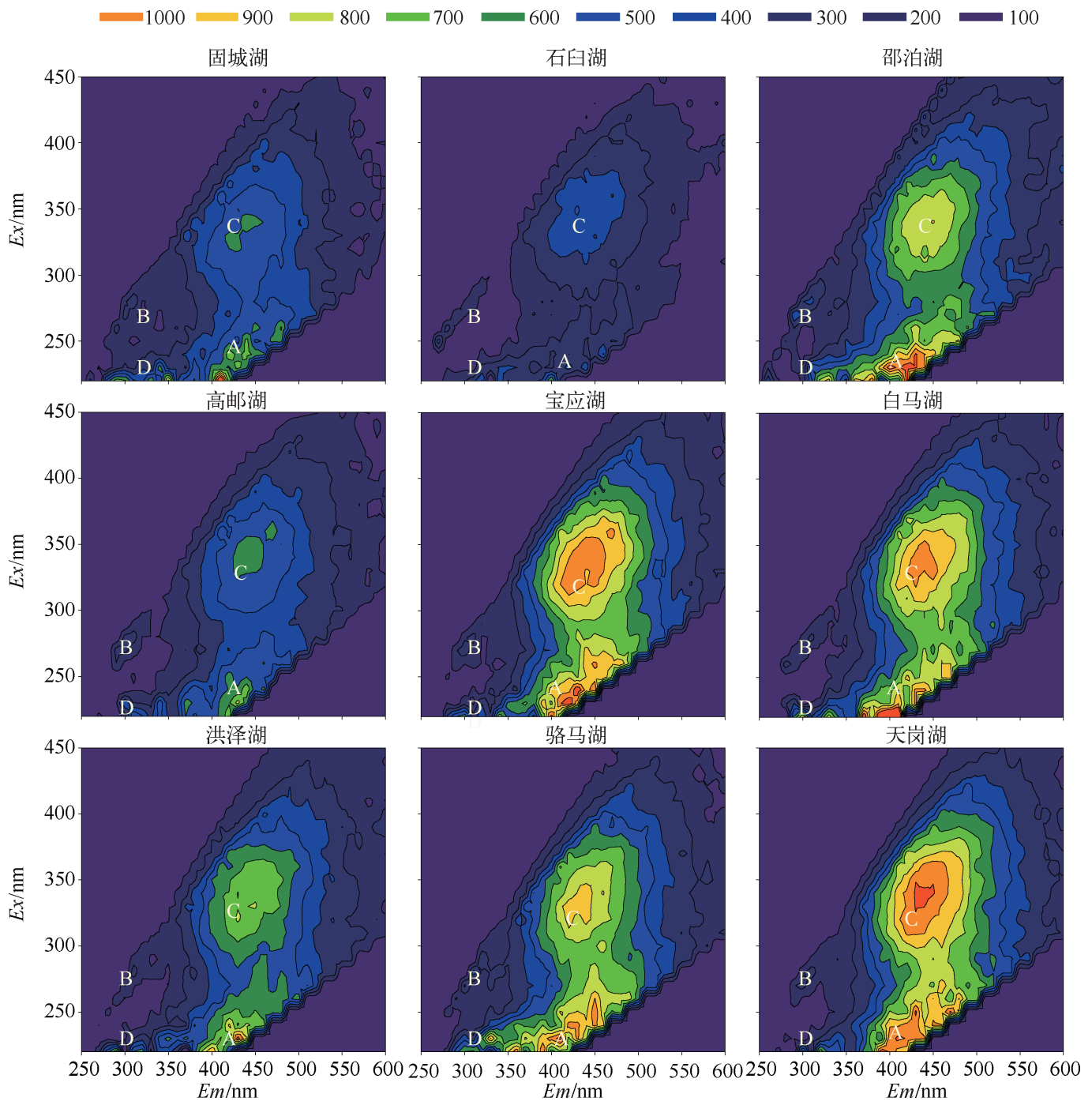

图 2 各个湖泊 DOM 三维苂光谱图

Fig. 2 Three-dimensional excitation emission matrix fluorescence spectroscopy (3DEEM)

of DOM in different lakes

380 和 $430 \mathrm{~nm}$ 处的比值, 它也可以用来估计湖泊内源物质对水体中 DOM 的相对贡献, 当 $B I X$ 值在 $0.8 \sim 1.0$ 时表明 DOM 由生物或微生物活动产生, 而低于 0.6 时则可认为 DOM 主要来自于外源 ${ }^{[40]}$. 本研究中, 各湖泊 的 $F I$ 和 $B I X$ 分别在 $1.13 \sim 1.30$ 和 $0.47 \sim 0.67$ 之间 (表 2), 体现出 DOM 极强的外源性.

不同来源 DOM 的紫外区与可见区类腐殖酸荧光强度比值 $r(\mathrm{~A}, \mathrm{C})$ 相差显著. Coble ${ }^{[11]}$ 研究河流 DOM 的 $r(\mathrm{~A}, \mathrm{C})$ 平均值为 1.08 , 国内有研究表明红枫湖 $r(\mathrm{~A}, \mathrm{C})$ 在 $1.76 \sim 1.97$ 之间 ${ }^{[41]}$, 而太湖则在 $1.12 \sim 2.36$ 之 间 ${ }^{[39]}$, 因此 $r(\mathrm{~A}, \mathrm{C})$ 值的范围可作为湖泊类腐殖酸物质来源差异性的指标. 本研究中, 各湖泊的 $r(\mathrm{~A}, \mathrm{C})$ 值在 $0.99 \sim 1.29$ 之间 (表 2), 其分布范围远小于太湖, 说明这些湖泊可能具有相似的类腐殖酸物质来源.

相关性分析可以判定不同湖泊 DOM 来源差异性. 紫外区类腐殖酸苂光主要由一些低分子量、高苂光效 率的有机物质所引起, 而可见区类腐殖酸苂光则是由相对稳定、高分子量的芳香性类富里酸物质所产生 ${ }^{[42]}$. 因此, 如果类腐殖酸菼光峰 $\mathrm{A}$ 和 $\mathrm{C}$ 的相对苂光强度存在较好的线性关系, 即可认为其来源具有相似性 ${ }^{[38]}$. 与此相似, 如果类蛋白物质与类腐殖酸物质的相对苂光强度具有相关性, 则可认为类蛋白物质和类腐殖酸 
表 3 单位 DOC 浓度下不同苂光峰强度的相关性

Tab. 3 The correlation of fluorescence intensities per unit organic carbon at different peaks

\begin{tabular}{ccccc}
\hline & $F I_{\mathrm{A}}$ & $F I_{\mathrm{B}}$ & $F I_{\mathrm{C}}$ & $F I_{\mathrm{D}}$ \\
\hline$F I_{\mathrm{A}}$ & 1 & & & \\
$F I_{\mathrm{B}}$ & $0.833^{* * 1)}$ & 1 & & \\
$F I_{\mathrm{C}}$ & $0.975^{* *}$ & $0.769^{* *}$ & 1 & \\
$F I_{\mathrm{D}}$ & $0.835^{* *}$ & $0.803^{* *}$ & $0.823^{* *}$ & 1 \\
\hline
\end{tabular}

1) $* *$ 显著性水平为 $P<0.01$ (双尾检验); $F I_{\mathrm{A}} \sim F I_{\mathrm{D}}$ 分别表示三维荧光光谱的 $\mathrm{A} \sim \mathrm{D}$ 峰苂光指数.
类物质具有相似的来源, 或者两者的结构之间 具有某种联系 ${ }^{[43]}$. 四个菼光峰的相对菼光强度 的相关性分析结果可以看出, 类蛋白菼光峰 (峰 $B$ 、峰 D) 之间、类腐殖酸苂光峰 (峰 A、峰 $C$ ) 之间 以及类蛋白苂光峰 (峰 $B$ 、峰 $D$ ) 与类腐殖酸苂光 峰( 峰 A、峰 C) 之间均存在良好的相关性 (表 3 ), 表明这些湖泊的类腐殖酸及类蛋白物质可 能存在相同的来源.

三维荧光图谱的多个指标均表明外源输人 是这些湖泊中 DOM 的主要来源. 湖泊 DOM 的 外源输人包括地表径流引起的湖泊周围土壤中 动植物碎屑及有机质的输人、生活污水及工农业污水的直接排人、养殖饲料投加以及人湖河流输人等 ${ }^{[39]}$. 生 活污水以及养殖饵料的分解物等均具有类富里酸含量低、类蛋白含量高的特点 ${ }^{[4]]}$, 但三维菼光图谱中类蛋 白苂光并不强烈, 表明这些因素对江苏西部湖泊中 DOM 的贡献甚小, 而江苏西部湖泊均为过水性湖泊,换 水周期短 ${ }^{[15]}$, 因此可推断人湖河流输人是这些湖泊 DOM 的主要来源. 固城湖和石臼湖属青七江、水阳江水 系,该流域主要以农业耕作为主 ${ }^{[45]}$. 洪泽湖等苏北湖泊属淮河水系,众所周知, 淮河流域总体上处于经济欠 发达地区,第一产业在沿淮四省的产业结构中仍占有较大比重 ${ }^{[46]}$. 农村地区的土壤、农田堆肥和腐烂植物中 的腐殖质会随雨水和灌溉水输人河道, 从而进人湖泊. 此外, 淮河流域占工业污染负荷 $50 \%$ 以上的企业是造 纸企业,其规模小、设备落后、治污难度大 ${ }^{[47]}$. 造纸废水中含有大量的纤维素、木质素等有机质,因此这可能 是苏北湖泊中 DOM 的另一个重要来源. 人湖河流携带是江苏西部湖泊 DOM 的主要来源, 同时也是这些湖 泊富营养化的重要因素 ${ }^{[16]}$, 因此应加强对江苏西部湖泊人湖河道的环境保护.

\section{3 结论}

1 ) 江苏省西部湖泊中 DOM 的紫外-可见吸收光谱的 $S U V A 、 E_{2} / E_{3} 、 E_{3} / E_{4}$ 和指数函数斜率均表明 DOM 的结构复杂度及分子量大小依次为郡伯湖 $>$ 天岗湖 $>$ 白马湖 $>$ 石臼湖 $>$ 洪泽湖 $>$ 固城湖 $>$ 骆马湖 $>$ 高邮 湖 > 宝应湖.

2) 江苏省西部各湖泊的 DOM 三维荧光图谱中 $F I$ 值和 $B I X$ 值分别在 $1.13 \sim 1.30$ 和 $0.47 \sim 0.67$ 之间, 显示 DOM 具有极强的外源性.

3) DOM 的 $r(\mathrm{~A}, \mathrm{C})$ 值及相关性研究结果均表明江苏西部湖泊中 DOM 具有相似的来源, 结合这些湖泊 流域的经济发展水平可推断人湖河流携带的由农业及其下游产业产生的有机质是江苏西部湖泊中 DOM 的 主要来源.

\section{4 参考文献}

［1］吴丰昌,王立英,黎 文等. 天然有机质及其在地表环境中的重要性. 湖泊科学,2008,20(1):1-12.

[ 2 ] Judd KE, Crump BC, Kling GW. Variation in dissolved organic matter controls bacterial production and community composition. Ecology, 2006, 87: 2068-2079.

[ 3 ] Mladenov N, Zheng Y, Miller MP et al. Dissolved organic matter sources and consequences for iron and arsenic mobilization in Bangladesh aquifers. Environ Sci Technol, 2010, 44: 123-128.

[ 4 ] Znachor P, Nedoma J. Importance of dissolved organic carbon for phytoplankton nutrition in a eutrophic reservoir. $J$ Plankton Res, 2010, 32(3) : 367-376.

[ 5 ] Peterson BJ. Stable isotopes as tracers of organic matter input and transfer in benthic food webs: A review. Acta Oecol, 1999, 20 (4) : 479-487.

[ 6 ] Troyer ID, Bouillonl S, Barker S et al. Stable isotope analysis of dissolved organic carbon in soil solutions using a catalytic combustion total organic carbon analyzer-isotope ratio mass spectrometer with a cryofocusing interface. Rapid Commun Mass Spectrom, 2010, 24 : 365-374. 
[ 7 ] Her N, Amy G, Chung J et al. Characterizing dissolved organic matter and evaluating associated nanofiltration membrane fouling. Chemosphere, 2008,70 : 495-502.

[ 8 ] Wu F, Tanoue E. Molecular mass distribution and fluorescence characteristics of dissolved organic ligands for copper ( II) in lake Biwa, Japan. Org Geochem, 2001, 32: 11-20.

[ 9 ] Woods G, Simpson M, Keleher B et al. Online high-performance size exclusion chromatography-nuclear magnetic resonance for the characterization of dissolved organic matter. Environ Sci Technol, 2010, 44: 624-630.

[10] Fu PQ, Wu FC, Liu CQ et al. Spectroscopic characterization and molecular weight distribution of dissolved organic matter in sediment porewaters from Lake Erhai, Southwest China. Biogeochemistry, 2006, 81 : 179-189.

[11] Coble PG. Characterization of marine and terrestrial DOM in seawater using excitation-emission matrix spectroscopy. Mar Chem, 1996, 51: 325-346.

[12 ] Hera N, Amya G, McKnighta D et al. Characterization of DOM as a function of MW by fluorescence EEM and HPLC-SEC using UVA, DOC, and fluorescence detection. Water Res, 2003, 37: 4295-4303.

[13] Wada S, Aokib MN, Tsuchiya Y et al. Quantitative and qualitative analyses of dissolved organic matter released from Ecklonia cava Kjellman, in Oura Bay, Shimoda, Izu Peninsula, Japan. J Exp Mar Biol Ecol, 2007, 349 : $344-358$.

[14] Parlanti E, Wörz K, Geoffroy L et al. Dissolved organic matter fluorescence spectroscopy as a tool to estimate biological activity in a coastal zone submitted to anthropogenic inputs. Org Geochem, 2000, 31: 1765-1781.

[15] 中国科学院南京地理研究所湖泊室. 江苏湖泊志. 南京: 江苏科学技术出版社, 1982 .

[16] 范成新,羊向东, 史龙新等. 江苏湖泊富营养化特征、成因及解决途径. 长江流域资源与环境, 2005, 14 (2) : $218-223$.

[17］章宗涉, 黄祥飞. 淡水浮游植物研究方法. 北京:科学出版社, 1991:338,340,345-347.

[18] Green S, Blough N. Optical absorption and fluorescence properties of chomophoric dissolved organic matter in natural waters. Limnol Oceanogr, 1994, 39(8) : 1903-1916.

[19] Komatsu K, Nakajima F, Furumai H et al. Characterization of dissolved organic matter (DOM) removal by iron coagulation using spectrofluorimetry and pyrolysis GC/MS analysis. J Wat Supply Res Technol-AQUA, 2005, 54(3) : 157-163.

[20] Zepp RG, Sheldon WM, Moran MA. Dissolved organic fluorophores in southeastern US coastal waters: correction methods for eliminating Rayleigh and Raman scattering peaks in excitation-emission matrices. Mar Chem, 2004, 89 : 15-36.

[21] Ohno T. Fluorescence inner-filtering correction for determining the humification index of dissolved organic matter. Environ Sci Technol, 2002,36 : 742-746.

[22] Weishaar J, Aiken G, Bergamaschi B et al. Evaluation of specific ultraviolet absorbance as an indicator of the chemical composition and reactivity of dissolved organic carbon. Environ Sci Technol, 2003, 37 : 4702-4708.

[23 ] Bricaud A, Morel A, Prieur L. Absorption by dissolved organic matter of the sea (yellow substance) in the UV and visible domains. Limnol Oceanogr, 1981, 26(1) : 43-53.

[24] Tipping E, Corbishley HT, Koprivnjak JF et al. Quantification of natural DOM from UV absorption at two wavelengths. Environ Chem, 2009, 6(6): 472-476.

[25] Buffle J, Deladoey P, Zumstein J et al. Analysis and characterization of natural organic matters in freshwaters. I. Study of analytical techniques. J Hydrol, 1983, 44(2) : 325-362.

[26] McKnight DM, Boyer EW, Westerhoff PK et al. Spectrofluorometric characterization of dissolved organic matter for indication of precursor organic materials and aromaticity. Limnol Oceanogr, 2001, 46(1) : 38-48.

[27] Chin YP, Aiken G, O'Loughlin E. Molecular weight, polydispersity, and spectroscopic properties of aquatic humic substances. Environ Sci Technol, 1994, 28: 1853-1858.

[28] Huovinen PS, Penttil H, Soimasuo MR. Spectral attenuation of solar ultraviolet radiation in humic lakes in Central Finland. Chemosphere, 2003, 51(3): 205-214.

[29] Artinger R, Buckau G, Geyer S et al. Characterization of groundwater humic substances: influence of sedimentary organic carbon. Appl Geochem, 2000, 15: 97-116.

[30 ] Peuravuori J, Pihlaja K. Isolation and characterization of natural organic matter from lake water: comparison of isolation with solid adsorption and tangential membrane filtration. Environ Int, 1997, 23(4) : 441-451.

[31] Abbt-Braun G, Lankes U, Frimmel FH. Structural characterization of aquatic humic substances: The need for a multiple method approach. Aquat Sci, 2004, 66: 151-170. 
[32] Helms JR, Stubbins A, Ritchie JD et al. Absorption spectral slopes and slope ratios as indicators of molecular weight, source, and photobleaching of chromophoric dissolved organic matter. Limnol Oceanogr, 2008, 53 (3) : 955-969.

[33 ] Tamara ECK, Anderson CA, Morgenstern K. Determining sources of dissolved organic carbon and disinfection byproduct precursors to the Mckenzie River, Oregon. J Environ Qual, 2010, 39: 2100-2112.

[34] Fichot CG, Benner R. A novel method to estimate DOC concentrations from CDOM absorption coefficients in coastal waters. Geophys Res Lett, 2011, 38( L03610, 5).

[35] Zhang YL, Liu ML, Qin BQ et al. Photochemical degradation of chromophoric-dissolved organic matter exposed to simulated UV-B and natural solar radiation. Hydrobiologia, 2009, 627 : 159-168.

[36] Nelson NB, Carlson CA, Steinberg DK. Production of chromophoric dissolved organic matter by Sargasso Sea microbes. Mar Chem, 2004, 89(1): 273-287.

[37] Romera-Castillo C, Sarmento H, Álvarez-Salgado XA et al. Production of chromophoric dissolved organic matter by marine phytoplankton. Limnol Oceanogr, 2010, 55(1) : 446-454.

[38] 刘明亮, 张运林, 秦伯强. 太湖人湖河口和开敞区 CDOM 吸收和三维苂光特征. 湖泊科学,2009,21(2):234-241.

[39] 宋晓娜, 于 涛, 张 远等. 利用三维荧光技术分析太湖水体溶解性有机质的分布特征及来源. 环境科学学报, $2010, \mathbf{3 0}(11): 2321-2331$.

[40 ] Huguet A, Vacher L, Relexans S et al. Properties of fluorescent dissolved organic matter in the Gironde Estuary. Org Geochem, 2009, 40: 706-719.

[41] 傅平青, 刘丛强, 吴丰昌. 溶解有机质的三维苂光光谱特征研究. 光谱学与光谱分析, 2005,25(12):2024-2028.

[42] Baker A, Curry M. Fluorescence of leachates from three contrasting landfills. Water Res, 2004, 38 : 2605-2613.

[43] 傅平青, 刘丛强, 吴丰昌等. 洱海沉积物孔隙水中溶解有机质的三维菼光光谱特征. 第四纪研究, 2004,24 (6): 695-700.

[44] Baker A, Ward D, Lieten SH et al. Measurement of protein-like fluorescence in river and waste water using a handheld spectrophotometer. Water Res, 2004, 38(12) : 2934-2938.

[45] 黄智华,薛 滨, 逢 勇. 江苏固城湖流域 1951-2000 年农业非点源氮, 磷输移的数值模拟研究. 第四纪研究, 2008,28 (4):674-682.

[46] 董广霞,毛剑英. 淮河流域污染“久治不愈” 原因浅析及治理措施建议. 中国环境监测, 2005,21(6):75-78.

[47] 吴舜泽,夏 青, 刘鸿亮. 中国流域水污染分析. 环境科学与技术,2000,23(2):1-6. 